\title{
Axon Initiation and Growth Cone Turning on Bound Protein Gradients
}

\author{
Junyu Mai, ${ }^{1}$ Lee Fok, ${ }^{1}$ Hongfeng Gao, ${ }^{2}$ Xiang Zhang,,${ }^{1,3}$ and Mu-ming Poo ${ }^{2}$ \\ ${ }^{1}$ National Science Foundation Nano-Scale Science and Engineering Center and ${ }^{2}$ Department of Molecular and Cell Biology, University of California, \\ Berkeley, and ${ }^{3}$ Materials Sciences Division, Lawrence Berkeley National Laboratory, Berkeley, California 94720
}

Extracellular gradients of secreted guidance factors are known to guide axon pathfinding and neuronal migration. These factors are likely to bind to cell surfaces or extracellular matrix, but whether and how they may act in bound gradients remains mostly unclear. In this study, we have developed a new technique for rapid production of stable microscopic gradients of substrate-bound proteins by covalent bonding of the proteins with an epoxy-coated glass substrate while they are diffusing in an agarose gel. Using this method, we found that bound gradients of netrin-1 and brain-derived neurotrophic factor (BDNF) can polarize the initiation and turning of axons in cultured hippocampal neurons. Furthermore, bound BDNF gradient caused attractive and repulsive polarizing response on gradients of low- and high-average density of BDNF, respectively. This novel bidirectional response to BDNF depended on the basal level of cAMP in the neuron. Finally, our data showed that the neuron's attractive response to bound BDNF gradient depended on the absolute difference rather than the relative difference in the BDNF density across the neuron, with a minimal effective difference of $1-2 \mathrm{BDNF}$ molecule/ $\mu \mathrm{m}^{2}$ on the substrate surface. Thus, substrate-bound guidance factors are highly effective in polarizing axon initiation and growth, and the diffusive printing technique is useful for studying neuronal responses induced by bound protein gradients.

\section{Introduction}

Extracellular gradients of secreted proteins play important roles in axon guidance (Tessier-Lavigne and Goodman, 1996; Dickson, 2002) and neuronal polarization (Arimura and Kaibuchi, 2007) and migration (Song and Poo, 2001). With a few exceptions (Baier and Bonhoeffer, 1992; Dertinger et al., 2002; Moore et al., 2006; von Philipsborn et al., 2006), neuronal responses to extracellular gradients were mostly studied for diffusible protein gradients established in solution or in three-dimensional gel matrices (Tessier-Lavigne et al., 1988; Lohof et al., 1992; Song et al., 1997; Keenan and Folch, 2008). However, most secreted proteins in intact tissues are likely to bind to cell surfaces or to the extracellular matrix (ECM) that fills the intercellular space (Nathan and Sporn, 1991; Kennedy et al., 2006), and must thus act on the target cell in a bound form. Binding to cell surfaces or ECM allows secreted growth factors to be maintained at a stable high concentration (Park et al., 1993), protected from proteolytic degradation (Saksela et al., 1988), and modulated for their interactions with target cells (Jones et al., 1993). However, it remains mostly unclear how bound gradients of secreted guidance factors influences neuronal development, and whether the immobiliza-

Received March 7, 2009; accepted April 7, 2009.

This work was supported by National Institutes of Health (NIH) through NIH Roadmap for Medical Research (PN2 EY018228) to X.Z., and by an NIH grant (NS22764) to M-m.P. J.M. was supported by a National Science Foundation Integrative Graduate Education and Research Traineeship.

Correspondence should be addressed to either of the following: Dr. Mu-ming Poo, Department of Molecular and Cell Biology, 221 Life Science Addition, University of California, Berkeley, CA 94720, E-mail: mpoo@berkeley.edu; or Dr. Xiang Zhang, Nano-Scale Science and Engineering Center, 3112 Etcheverry Hall, University of California, Berkeley, CA 94720, E-mail: xiang@berkeley.edu.

DOI:10.1523/JNEUROSCI.1121-09.2009

Copyright $\odot 2009$ Society for Neuroscience $\quad$ 0270-6474/09/297450-09\$15.00/0 tion of the factors and their membrane receptors by the bound gradients may yield effects that are distinct from those induced by gradient of these factors in the diffusible form.

The difficulty of investigating the effects of bound gradients lies in generating microscopic substrate-bound protein gradients suitable for in vitro neuronal culture and analysis. After decades of research on gradient surfaces (Genzer and Bhat, 2008), some sophisticated methods have been developed. These systems (Baier and Bonhoeffer, 1992; Dertinger et al., 2002; Moore et al., 2006) mostly required sophisticated device fabrication and experimental set-up, large amounts of protein, and relatively long preparation time. Another novel method based on contact printing (von Philipsborn et al., 2006) produced digitized, not continuous bound protein gradients. In this study, we have developed a new technique, termed "diffusive printing," that allows rapid production of well controlled substrate-bound protein gradients. This method uses a removable hydrogel matrix stamp in contact with an epoxy-coated glass coverslip (see Fig. 1a). The protein solution is first delivered into the miniature source channels prefabricated at the bottom of the stamp. After a defined duration that allows the establishment of the intended diffusible protein gradient in the matrix and the printing of proteins onto the substrate surface by covalent bonding, the stamp is removed from the glass substrate and stable bound gradients of protein are obtained. Hundreds of bound protein gradients can be produced in parallel within one operation. This method was then used for studying the effects of bound gradients of two known axon guidance factors, netrin-1 and brain-derived neurotrophic factor (BDNF), on neuronal development. We found that bound gradients of these molecules are highly effective in polarizing the differentiation of the axon and inducing chemotropic turning of 
a
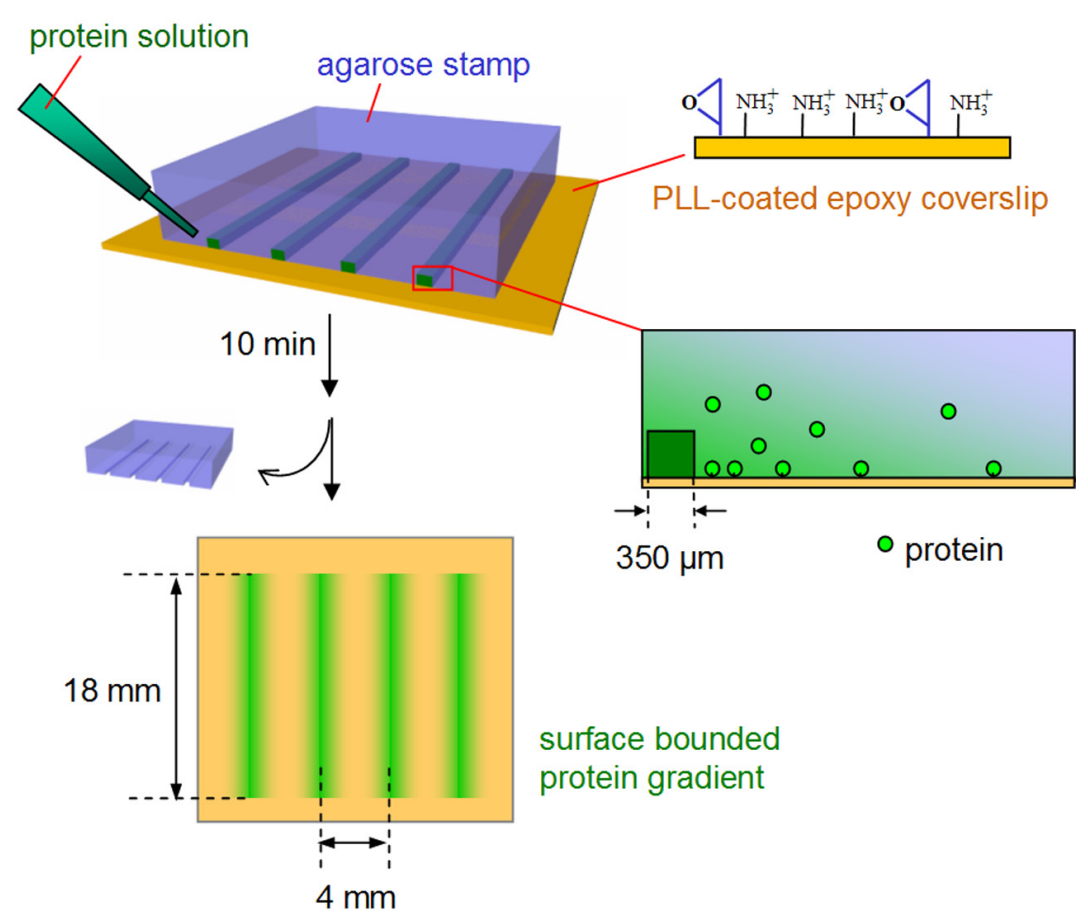

surface bounded

protein gradient

b
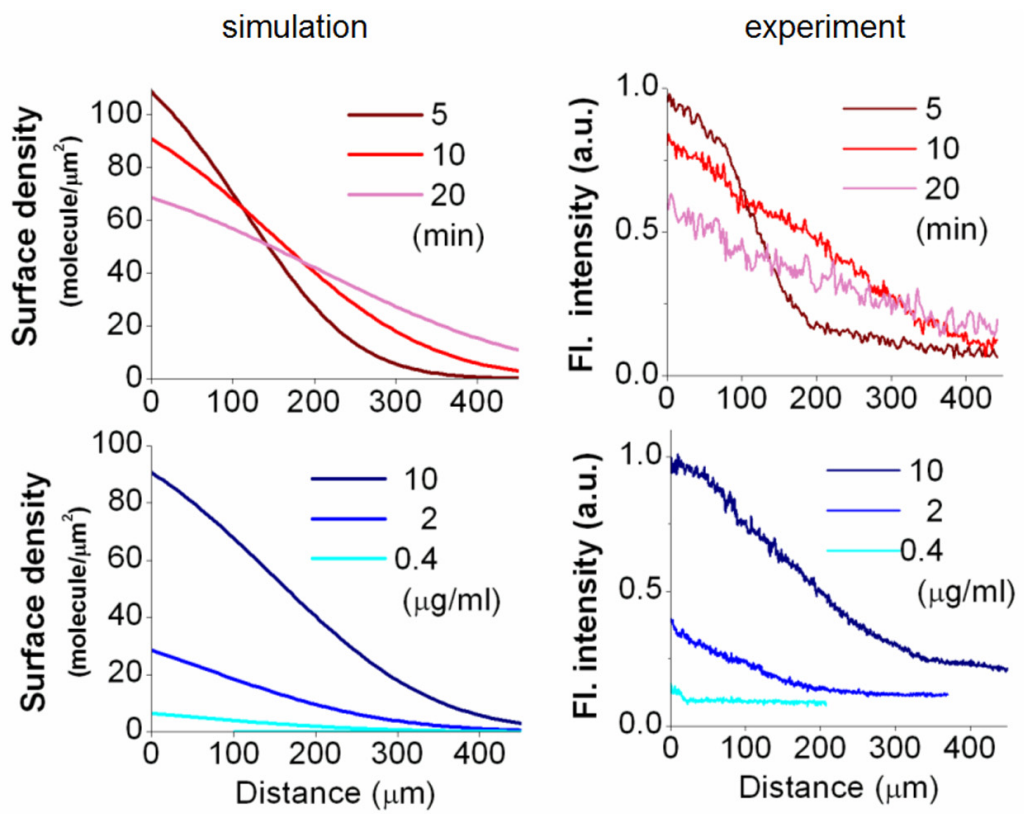

Figure 1. Diffusive printing method for generating surface-bound protein gradients. $\boldsymbol{a}$, Schematic diagrams of the diffusive printing process. An $18 \times 18 \times 10 \mathrm{~mm}$ agarose gel stamp, with four channels (each $350 \mu \mathrm{m}$ wide, $100 \mu \mathrm{m}$ deep, and $4 \mathrm{~mm}$ apart) fabricated at the bottom, was put on a PLL-coated epoxy glass coverslip. Protein solution was delivered into the channels using a pipette. The agarose stamp was later removed from the coverslip after a defined printing time (5-30 min). Eight stripes of bound protein gradient were produced on each coverslip. $\boldsymbol{b}$, Comparison of computer-simulated gradient profiles (left panels) and experimentally measured fluorescent intensity profiles (right panels) of bound FITC-IgG gradient. Bound gradients of FITC-IgG were generated by diffusive printing using $5 \%$ agarose gel, with $10 \mu \mathrm{g} / \mathrm{mI} \mathrm{FITC-IgG} \mathrm{and} \mathrm{different} \mathrm{printing} \mathrm{times} \mathrm{(top} \mathrm{panels),} \mathrm{or}$ with 10-min printing time and different concentration of FITC-IgG (bottom panels).

the axonal growth cones in cultured hippocampal neurons. We also observed and quantitatively analyzed a novel form of surface BDNF density-dependent bidirectional responses and the minimal BDNF surface gradient required for inducing polarized axon initiation and axonal growth cone turning.

\section{Materials and Methods}

Diffusive printing method for creating bound protein gradient

Silicon mold. A mask of parallel black lines, each $350 \mu \mathrm{m}$ in width and $4 \mathrm{~mm}$ apart, was designed in L-Edit (Tanner Research) and printed on a transparent plastic sheet using a highresolution (0.125 mil) laser plotter (ArtNetPro). A $2-\mu \mathrm{m}$-thick layer of positive photoresist S1818 (Shipley) was spin coated at $15,000 \mathrm{rpm}$ for $30 \mathrm{~s}$ on a 4 inch silicon wafer. The pattern was transferred from the transparent mask to the silicon wafer using standard photolithography (Quintel) and $100 \mu \mathrm{m}$ deep reactive dry etching (Surface Technology System). Etched wafer was plasma treated to remove photoresist layer and then vapor-coated in a vacuum bowl for $10 \mathrm{~h}$ with [tridecafluoro-1,1,2,2tetrahydrooctyl]-1-trichlorosilane (United Chemical Technologies) to make the surface hydrophobic, a necessary step to ensure a smooth gradient and easy removal of the agarose gel from the wafer later. Before use, we sterilized the wafer with $95 \%$ ethanol and rinse with deionized water, and dried the wafer with filtered air inside a biological safety cabinet.

Agarose stamp. For each wafer, $5 \mathrm{~g}$ of agarose (Invitrogen) was thoroughly stirred in $100 \mathrm{ml}$ of PBS (Invitrogen) for $10 \mathrm{~min}$, autoclaved for $30 \mathrm{~min}$, and then gently poured onto the sterilized silicon wafer in a $150 \mathrm{~mm}$ Petri dish and kept at room temperature in a sterilized cabinet for $1 \mathrm{~h}$. After the gel solidified, it was detached from the silicon wafer and moved to a sterile Petri dish, and cut into $18 \times 18 \mathrm{~mm}$ stamps, each containing four channels at the bottom. These stamps were either used immediately or stored in small amount of PBS in a Parafilmsealed sterile Petri dish.

Epoxy coverslips. Before diffusive printing, we incubated epoxy-coated coverslips $(22 \times 22 \times$ $0.17 \mathrm{~mm}$, Arrayit SuperEpoxy2, $5 \times 10^{12}$ reactive group $/ \mathrm{mm}^{2}$ ) with $0.5 \mathrm{mg} / \mathrm{ml}$ poly-L-lysine (PLL) for at least $10 \mathrm{~h}$.

Diffusive printing. The PLL-coated coverslips was rinsed with deionized water and dried completely in filtered air. Excess PBS around the agarose stamps and inside the channels were thoroughly cleared by aspiration with a pipette. The stamps were then placed on top of each epoxy coverslip. We carefully delivered protein solution into the channels under the stamp using a $10 \mu \mathrm{l}$ pipette. The channels became invisible once they are filled with the liquid. Within $\sim 2$ $\mathrm{min}$, protein solution was absorbed by the agarose gel and the channels became clear again. After 10 min or other defined durations, we quickly removed the stamp from the coverslip. The patterned coverslip were kept dry at room temperature for $\sim 30$ min to ensure complete covalent reactions and then rinsed with $\mathrm{PBS}$ twice.

\section{Measurements of protein density on epoxy coverslips}

To calibrate the surface-bound protein density from the fluorescence intensity measurements, we made rectangular calibration channels, each $100 \mu \mathrm{m}$ in height and $2 \mathrm{~mm}$ in width, using double sided adhesive tapes and glass coverslips. A solution containing FITC-conjugated IgG (FITCIgG) (Sigma Aldrich; 4 FITC molecule/IgG) of a specific concentration 

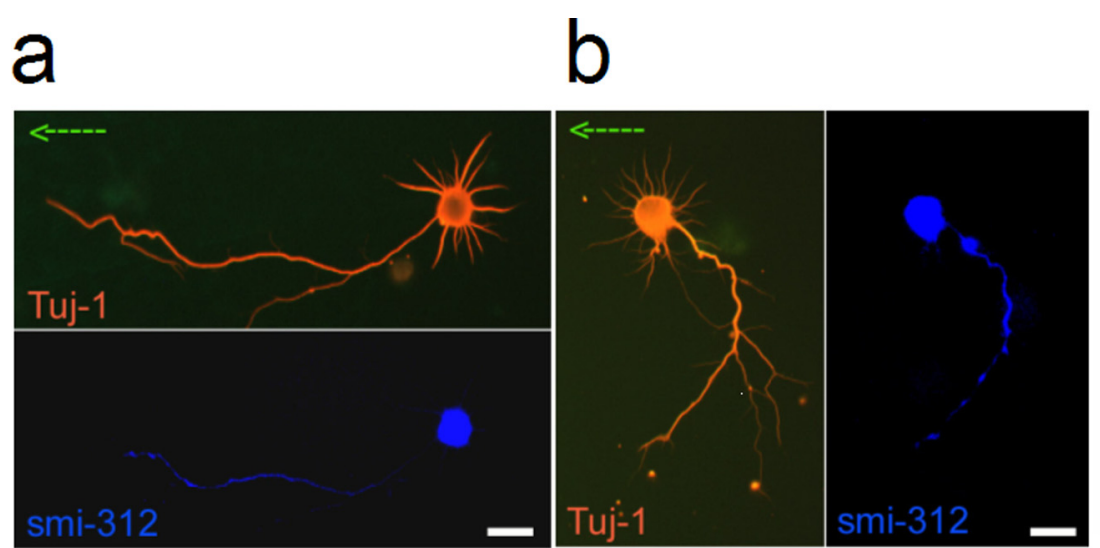

C

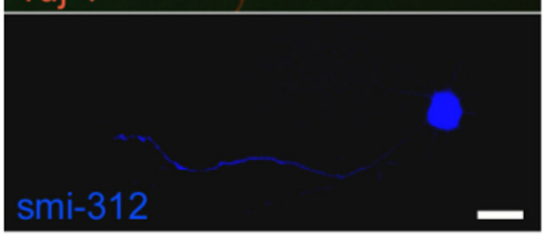

\section{b}
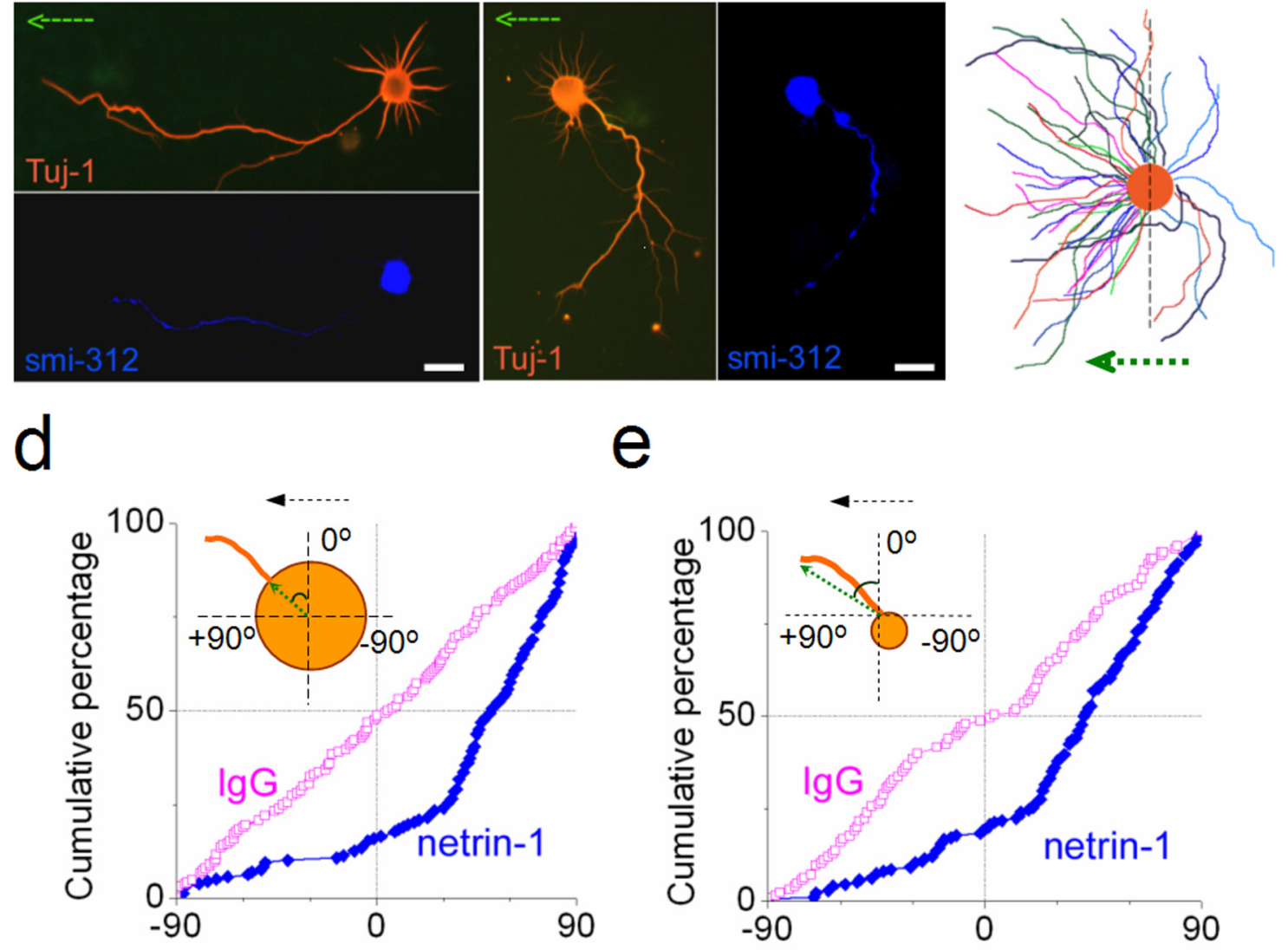

Axon initiation angle (deg)

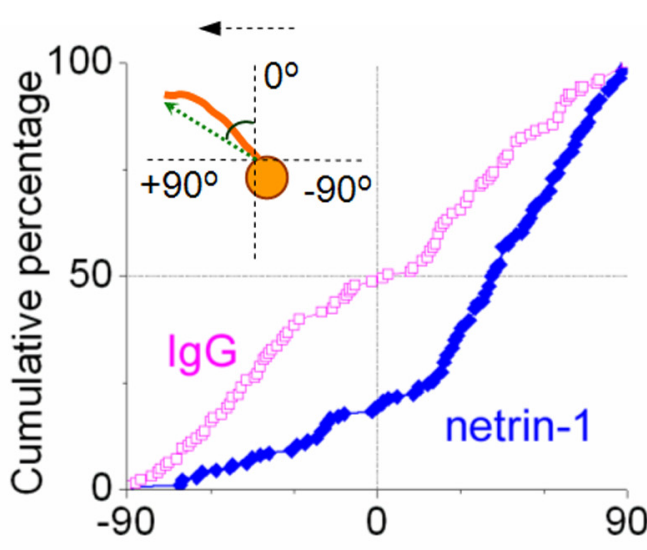

Axon turning angle (deg)

Figure 2. Axon initiation and growth cone turning on bound netrin-1 gradients. Hippocampal neurons were cultured for $40 \mathrm{~h}$ on bound netrin-1 gradient created by 10 -min diffusive printing using $100 \mu \mathrm{g} / \mathrm{ml}$ netrin-1. $\boldsymbol{a}, \boldsymbol{b}$, Images of two representative neurons immunostained for neuron- and axon-specific markers Tuj-1 and smi-312, respectively. Scale bars, $20 \mu \mathrm{m}$. c, Composite tracing of 40 randomly sampled axons of polarized hippocampal neurons (coded with color at random). The circle represents the soma. Arrows indicate increasing density of netrin-1.d, $e$, Cumulative percentage plots for the distribution of axon initiation angles $(\boldsymbol{d})$ and axon turning angles $(\boldsymbol{e})$ for all sampled single-axon neurons cultured on bound netrin-1 gradient and on control bound lgG gradient (10 min diffusive printing with $20 \mu \mathrm{g} / \mathrm{ml} \mathrm{FITC-IgG).} \mathrm{Insets,} \mathrm{Definition} \mathrm{of} \mathrm{the} \mathrm{axon} \mathrm{initiation} \mathrm{and} \mathrm{turning} \mathrm{angles.} \mathrm{The} \mathrm{angles} \mathrm{varied} \mathrm{from}+90^{\circ}$ to $-90^{\circ}$, with the positive and negative value indicating that the axon was initiated or turned toward the high- and low-density side of the gradient, respectively. The data for control lgG gradient $(n=128,3$ cultures) were significantly different from those for netrin-1 gradient ( $n=174,3$ cultures) $(p<0.001$, Kolmogorov-Smirnov test).

was loaded into the channels, and fluorescence intensity of the solution in the middle of the channel was measured using an epi-fluorescence microscope with a $40 \times$ objective lens [numerical aperture (NA) 1.3] and $500 \mathrm{~ms}$ single exposure that minimizes photobleaching. The number of FITC-IgG molecules that contributed to the measured fluorescence intensity was estimated from the protein concentration and the point spread function of the objective lens. The point spread function in the $z$-direction was approximated by a Gaussian function: $I=I_{\mathrm{o}} \exp \left[\left(-z^{2}\right) /\right.$ $\left(2 \sigma^{2}\right)$ ], where the half-width $\sigma$ is $0.75 \mu \mathrm{m}$ for an objective lens with NA 1.3 (Goldman and Spector, 2004), and $z$ is the distance between the fluorophores and the focal plane. The measured fluorescence intensity inside the calibration channel can be calculated by integrating all the fluorophore intensity in two three-dimensional cones, above and below the focal plane, as given by the following:

$$
2 \cdot \int_{0}^{50 \mu m} c \cdot I \cdot \pi \frac{z^{2}}{3} d z=2.5 \cdot \sigma^{3} \cdot c \cdot I_{0},
$$

where $c$ is the concentration of the fluorophores. The area covered by the point spread function on the $\mathrm{x}-\mathrm{y}$ plane was approximated to be $\sim 1 \mu \mathrm{m}^{2}$ for the same NA 1.3 lens. The equivalent section depth was then esti- mated to be $2.5 \cdot \sigma^{3} / 1 \mu \mathrm{m}^{2}=2 \mu \mathrm{m}^{2}$. This calculation demonstrates that we can assume the measured fluorescence intensity is equivalent to that contributed by fluorophores within a $2-\mu \mathrm{m}$-wide layer centered at the focus plane, and the fluorescence from other regions in the channel can be ignored. Using solutions of different concentrations of FITC-IgG in the calibration channel, we obtained the plot of measured fluorescent intensity versus the estimated FITC-IgG molecules per unit area of 1 $\mu \mathrm{m}^{2}$ within the calibration channel (supplemental Fig. S2, available at www.jneurosci.org as supplemental material). This calibration was used for converting fluorescence intensity to surface density of bound proteins on the epoxy coverslip. For estimating the BDNF density on the coverslip, as revealed by using polyclonal rabbit anti-BDNF antibody (PeproTech) and FITC-conjugated goat anti-rabbit polyclonal IgG (Sigma Aldrich), an additional multiplication factor of 1.6 was necessary to fit the experimental data with the simulated density profiles. The factor 1.6 may represent the average number of FITC-conjugated secondary antibody linked to each BDNF molecule on the substrate surface.

\section{Culturing and imaging hippocampal neurons}

The gradient-patterned coverslips were incubated in the PLL solution for an additional $4-5 \mathrm{~h}$ before cell plating. Cultures of dissociated hippocampal neurons were prepared from embryonic day 18 (E18) rats as previously described (Dotti et al., 1988) and were incubated in neuro- 
basal medium (Invitrogen) supplemented with B-27 (Invitrogen). As indicated, Sp-8-Br-cAMP (12.5 $\mu \mathrm{M}$, Axxora) or KT5720 (1 $\mu \mathrm{M}$ or $200 \mathrm{nM}$, EMD) was added into the culture medium $2 \mathrm{~h}$ after cell plating. For control experiments, function-blocking mouse monoclonal anti-DCC antibody [AF5] ( $2 \mu \mathrm{g} / \mathrm{ml}$, Abcam, AB16793) or mouse polyclonal antiTrkB antibody ( $2 \mu \mathrm{g} / \mathrm{ml}$, R\&D Systems, AF1494) were bath-applied during cell plating. After $40 \mathrm{~h}$ of culturing, neurons were fixed with $4 \%$ formaldehyde in PBS for $15 \mathrm{~min}$, and then permeabilized with $0.1 \%$ Triton X-100. For neuron and axon-specific immunostaining, cells were blocked for $0.5 \mathrm{~h}$ with 3\% IgG-free BSA (Jackson ImmunoResearch) and then stained for 2-24 $\mathrm{h}$ with rabbit monoclonal antibody against neuronal class III $\beta$-tubulin (Tuj-1, Covance) and with mouse monoclonal antibody against pan-axonal neurofilament marker (smi-312, Covance), both at 1:500 dilution. Alexa Fluor 568 Donkey anti-rabbit IgG (Invitrogen) and Alexa Fluor350 goat anti-mouse IgG (Invitrogen) were used as secondary antibodies in the subsequent $2 \mathrm{~h}$ incubation. After rinsing with PBS, coverslips were then mounted with Slowfade mounting media (Invitrogen) and sealed with nail polisher. All fluorescent images were taken from fluorescent microscope Axioimager (Zeiss), using a digital chargecoupled-device (CCD) 8-bit color camera (Qimaging) and a 40× NA1.3 oil-immersion objective lens (Zeiss). Fluorescent images were acquired using appropriate filters and iVision image processing software (BioVision Technologies).

Measurement of preference index of axon initiation and turning Axon initiation and turning angles were measured from cultured hippocampal neurons as defined in Figure 2, $d$ and $e$. Preference index (PI) of axon initiation (or turning) was calculated by the following formula: $\mathrm{PI}=(p-n) /(p+n)$, where $p$ and $n$ are the number of cells with positive and negative axon initiation (or turning) angles, respectively. A PI value varies from -1 , when all the cells have axons initiated from the side of the soma (or turned toward the side) facing the lower BDNF density, to +1 , when all the cells have axons initiated from (or turned toward) the other side. Averaged PI values are calculated from the PI values measured in three independent experiments performed for the same condition, with $50-60$ cells sampled in each experiment.

\section{Results}

\section{Gradient generation and characterization}

We first used FITC-IgG as a model protein to characterize the system. Using 5\% agarose gel as the matrix stamp, PLL-coated epoxy glass coverslips as the substrate, and $0.5 \mu \mathrm{l}$ of protein solution $(10 \mu \mathrm{g} / \mathrm{ml})$ in each of the four source channels $(350 \mu \mathrm{m}$ wide, $100 \mu \mathrm{m}$ deep, $18 \mathrm{~mm}$ long, and $4 \mathrm{~mm}$ apart), we produced eight stripes of bound FITC-IgG gradient on a $22 \times 22 \mathrm{~mm}$ coverslip after $10 \mathrm{~min}$ of diffusion time, with each gradient covering a distance of $\sim 500 \mu \mathrm{m}$ (Fig. 1a). The resulting protein gradient on the epoxy coverslip was stable in physiological solution for at least $48 \mathrm{~h}$ (supplemental Fig. S1, available at www. jneurosci.org as supplemental material). We chose epoxy coverslips because epoxy group can form irreversible covalent bonding with multiple nucleophilic groups on the protein surface, and the multipoint covalent immobilization helps to maintain the native conformation and activity of the bound protein (Mateo et al., 2000a). We found that precoating the epoxy coverslips with PLL enhanced protein binding to the epoxy surface, presumably by promoting its adsorption and subsequent covalent reaction with the epoxides, similar to the benefit provided by other heterofunctional epoxy substrates (Mateo et al., 2000b).

To theoretically predict the gradient profile, we numerically simulated the process of gradient formation (Fig. $1 b$; supplemental Information, available at www.jneurosci.org). In parallel, we experimentally measured the gradient profiles of bound IgG produced by diffusive printing and found that the measured IgG gradients matched well with the profiles predicted by simulations, in terms of the overall shape, distance covered, and relative

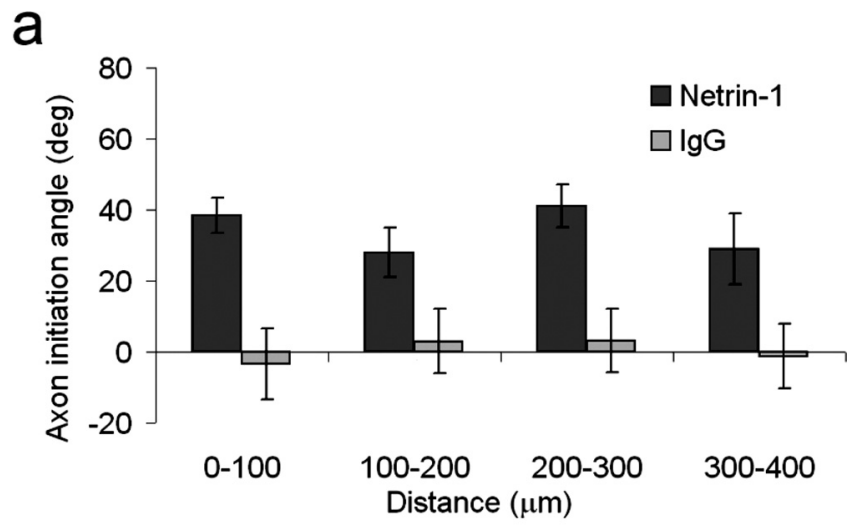

b

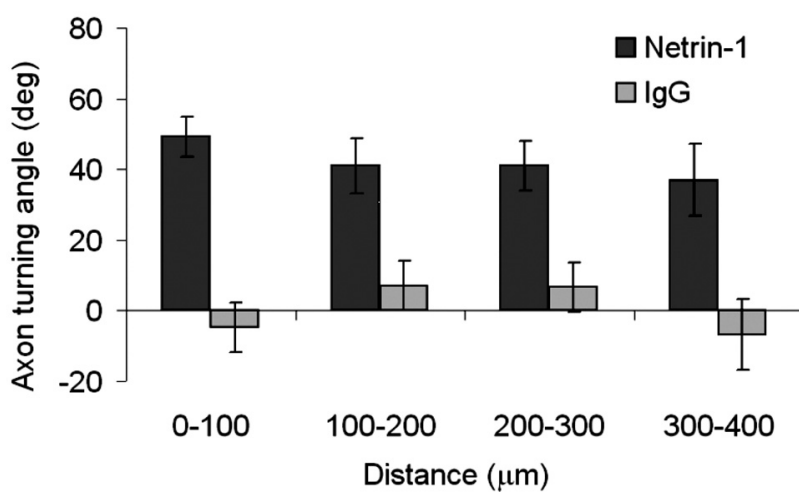

Figure 3. Axon initiation and turning at different distances along the bound netrin-1 gradient. $\boldsymbol{a}, \boldsymbol{b}$, Average axon initiation angle $(\boldsymbol{a})$ and axon turning angle $(\boldsymbol{b})$ were measured from neurons with soma located in each $100 \mu \mathrm{m}$-wide region along the bound gradient of netrin-1 and lgG, created by 10 min diffusive printing with $100 \mu \mathrm{g} / \mathrm{ml}$ netrin- 1 and $20 \mu \mathrm{g} / \mathrm{ml} \mathrm{FITC-lgG,}$ respectively (for netrin-1, $n=22-58$ in each region, 3 cultures; for $\lg G, n=24-37$ in each region, 3 cultures). Same set of data as that shown in Figure 2. Responses of axons to netrin-1 gradient were significantly different from those on IgG gradient ( $p<0.001$, KolmogorovSmirnov tests). Error bars indicate SEM.

maximal surface protein density (Fig. $1 b$ ). The maximal protein density increased with the concentration of the protein solution in the source channel, whereas for a given concentration, the maximal density and the slope of the gradient decreased with the diffusion time.

\section{Axon initiation and turning on bound netrin-1 gradient}

The process of spontaneous polarization of cultured hippocampal neurons plated on uniform substrates has been well characterized by Dotti et al. (1988). Within hours after plating, several morphologically equivalent neurites emerge from the soma. The polarization begins when one of the neurites exhibits accelerated growth and differentiates into the axon, while all other neurites become dendrites. To examine the effect of bound protein gradients on axon initiation and its pathfinding, we first focused on netrin-1, a secreted protein that serves as a chemoattractant for growing commissural axons in the spinal cord (Serafini et al., 1996) and a factor required for neuronal polarization in C. elegans (Adler et al., 2006). We created bound gradients of netrin-1 by $10 \mathrm{~min}$ diffusive printing using $100 \mu \mathrm{g} / \mathrm{ml}$ netrin-1 source solution, with the gradient direction revealed by an inert coprinted fluorescent protein FITC-conjugated bovine serum albumin (FITC-BSA, $5 \mu \mathrm{g} / \mathrm{ml}$ ). Before cell culturing, the printed substrate was incubated in a PLL solution for another $4-5 \mathrm{~h}$ to block the remaining epoxy groups. Dissociated hippocampal 
neurons prepared from E18 rats were then plated onto the substrate surface and examined $40 \mathrm{~h}$ later by immunostaining with neuron- and axon-specific markers Tuj-1 and smi-312, respectively. As shown by the example neurons (Fig. 2a,b) and by the composite tracings of a group of randomly sampled axons (Fig. 2c), more axons were found to emerge from the soma facing the side of higher netrin-1 density, and axon growth cones also exhibited marked chemoattractive turning toward the high density side. Since time-lapse recordings of live neurons over a $10 \mathrm{~h}$ period revealed that soma remained stationary, and the initiation site of the neurites on the somatic surface did not move significantly (data not shown), the apparent polarized axon initiation observed at $40 \mathrm{~h}$ after plating indicates that the netrin-1 gradient had triggered preferential axon differentiation of neurites that were initiated from the high netrin-1 side of the soma. Furthermore, axons growing on the bound gradient of netrin- 1 also exhibited chemotropic turning toward the high netrin-1 side of the gradient. These findings were confirmed by quantitative analysis of the angular distribution of the axon initiation site on the soma (Fig. $2 d$ ) and the final growth cone position (Fig. 2e) with respect to the direction of the gradient, for all polarized neurons exhibiting a single axon and multiple dendrites. When the data were analyzed based on the soma location in the bound netrin-1 gradient, we found no significant difference in the polarization effects of netrin-1 for neurons at different distances $>400 \mu \mathrm{m}$ along the gradient (Fig. 3). To examine whether the preference of axon initiation and turning is caused by activation of the specific netrin-1 receptor Deleted in Colorectal Cancer (DCC), rather than nonspecific interactions (e.g., adhesion) of the cell surface with the substrate-bound netrin-1, we bath applied the function-blocking anti-

body of DCC [monoclonal anti-DCC antibody AF5 (de la Torre et al., 1997; Ming et al., 1997)] to the culture, and found that neurite growth was greatly reduced and the polarization effect of the bound netrin- 1 was absent (initiation PI $=-0.05 \pm 0.03$; turning PI $=0.01 \pm 0.03$ ). The consistent effects of the bound netrin-1 gradient on both axon initiation and growth cone turning support the notion that secreted netrin-1 can exert polarizing and chemoattractive actions in a bound form in vivo, as suggested by previous immunohistochemical studies (Kennedy et al., 2006).

\section{Bidirectional axon initiation and turning on bound BDNF gradient}

Secreted growth factors of the neurotrophin family are known to exert chemotropic effects on growing axons both in vitro (Gundersen and Barrett, 1980; Song et al., 1997) and in vivo (Gillespie, b

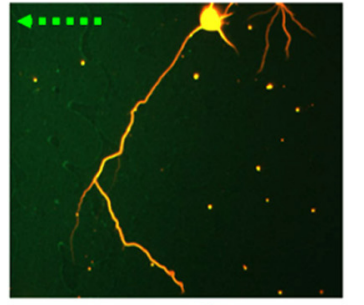

Q
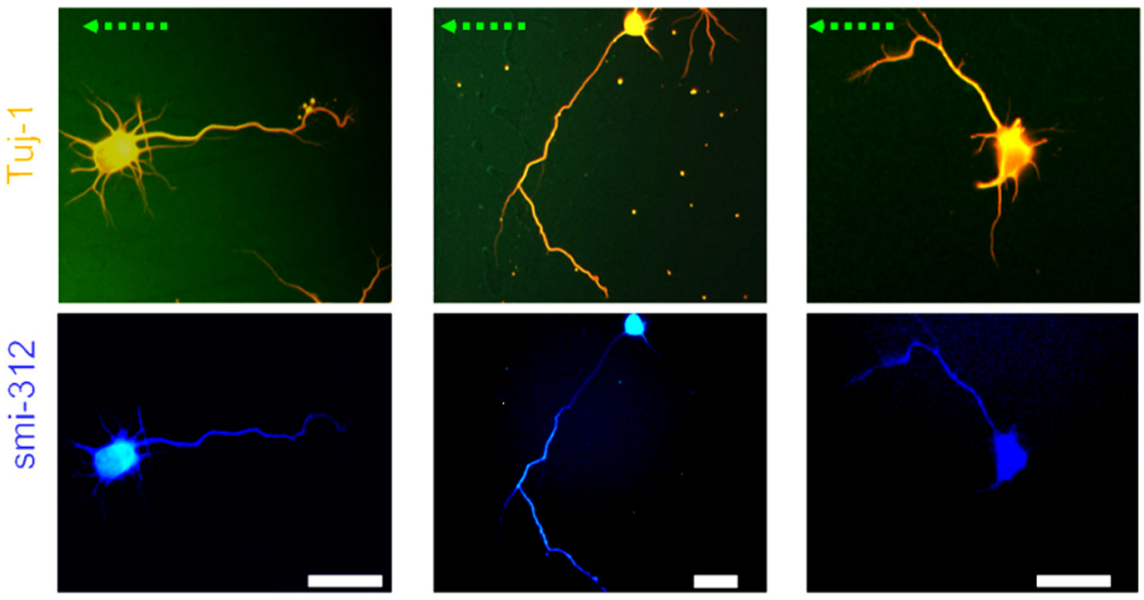

e

f

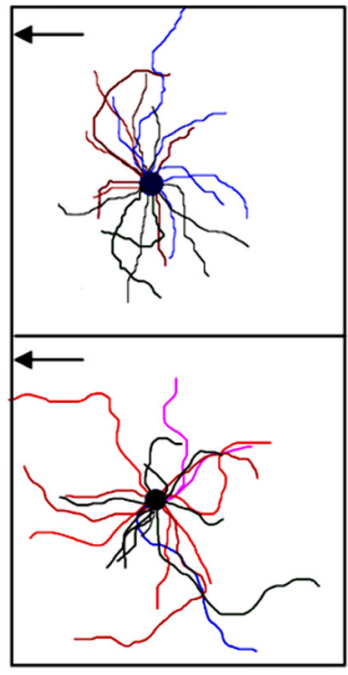

50-100 $\mu \mathrm{m}$

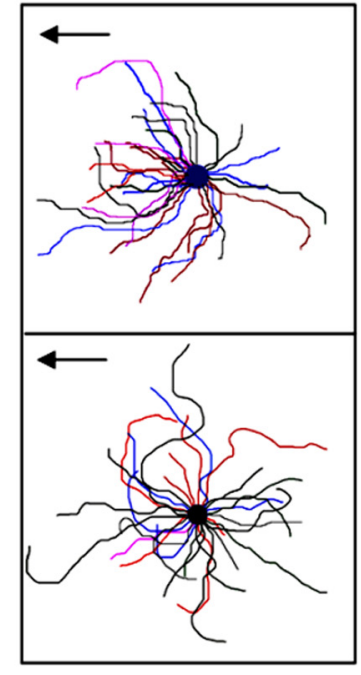

$100-300 \mu \mathrm{m}$
Figure 4. Bi-directional axon initiation and growth cone turning on bound BDNF gradient. Hippocampal neurons were culor $40 \mathrm{~h}$ on bound BDNF gradient created by $10 \mathrm{~min}$ diffusive printing with $20 \mu \mathrm{g} / \mathrm{ml}$ source BDNF. $\boldsymbol{a}-\boldsymbol{c}$, Images of neurons immunostained for neuron- and axon-specific markers Tuj-1 and smi-312, respectively, with the soma located $0-50 \mu \mathrm{m}(\boldsymbol{a})$, $-100 \mu \mathrm{m}(\boldsymbol{b})$, and 100-300 $\mu \mathrm{m}(\boldsymbol{c})$ from the side of highest BDNF density. Arrows indicate increasing BDNF density. Scale gradient (top panels), and along control lgG gradient produced with $20 \mu \mathrm{g} / \mathrm{ml}$ source lgG (bottom panels). For clarity, axons were randomly assigned with different colors. Arrows indicate increasing BNDF or lgG density.

2003; Genç et al., 2004). In the present study, we quantitatively examined the effect of various bound BDNF gradients on neuronal polarization and axon growth. Bound BDNF gradients were produced on PLL-treated epoxy glass surface by diffusive printing for $10 \mathrm{~min}$ with $20 \mu \mathrm{g} / \mathrm{ml}$ BDNF. Interestingly, we found that axons exhibited either attractive or repulsive responses, depending on their location along the BDNF gradient (Fig. 4). For neurons located within a distance of $\sim 50 \mu \mathrm{m}$ from the side of highest BDNF density, axons showed initiation and turning away from high BDNF (Fig. 4a,d), whereas those located between 100 and $300 \mu \mathrm{m}$ distances showed initiation and turning toward high BDNF (Fig. 4c,f). Between 50 and $100 \mu \mathrm{m}$, axons displayed heterogeneous responses, with apparent random axon initiation (Fig. 4e). Some axons showed initial extension toward the highdensity BDNF, but later turned or branched away from it (Fig. $4 b$ ). These findings were confirmed by quantitative analysis of the 
a

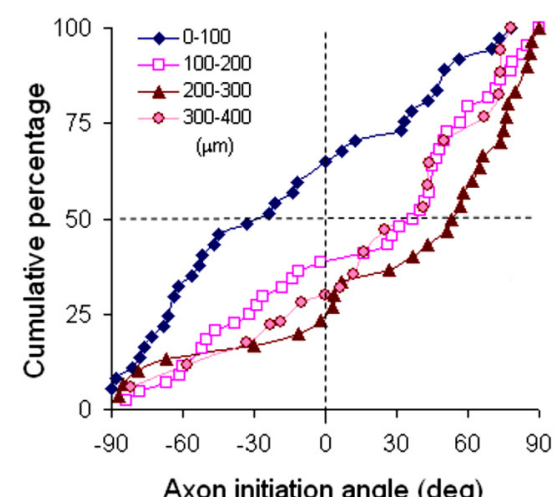

C

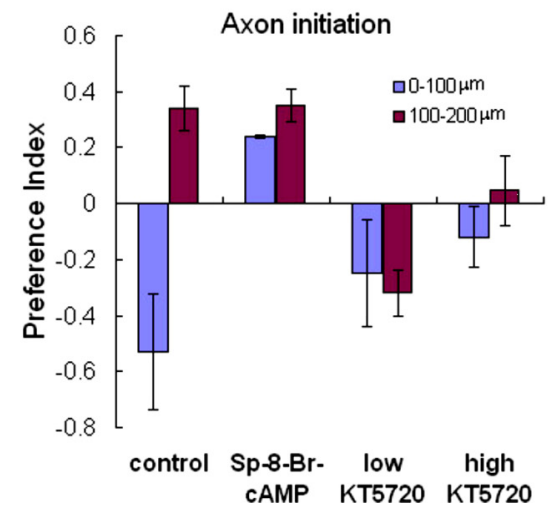

b

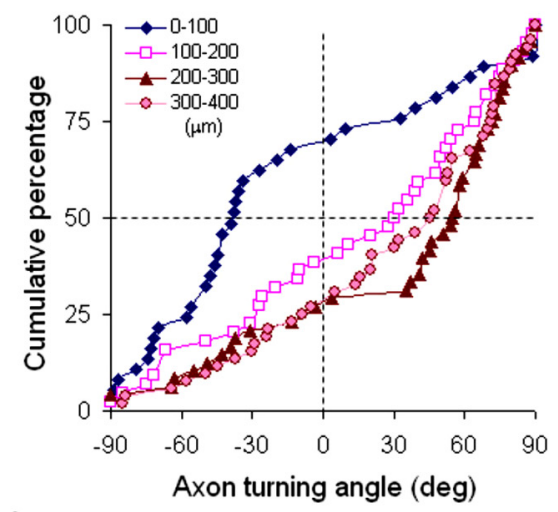

d

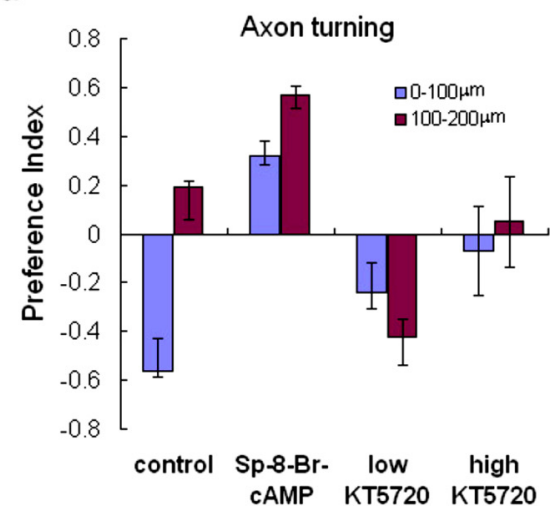

the cytosolic cAMP activity. Using preference index to characterize axon initiation and turning for the cells located in the $0-100 \mu \mathrm{m}$ and $100-200 \mu \mathrm{m}$ regions, we found that bath application of a membrane-permeable cAMP analog, Sp8-Br-cAMP $(12.5 \mu \mathrm{M})$, switched the axon's initiation and turning response from repulsion to attraction in the high-density region $(0-100 \mu \mathrm{m})$ of the BDNF gradient (Fig. $5 c, d$ ), whereas bath application of 200 nM KT5720, a specific protein kinase A (PKA) inhibitor, switched the axon's response from attraction to repulsion in the $100-200 \mu \mathrm{m}$ region (Fig. 5c,d). Both results suggest that the repulsive response at the high-density region may be attributed to a low cAMP level resulting from the high-level BDNF signaling. Furthermore, we found that activity of PKA is required for the polarization responses, because bath application of a higher concentration of KT5720 (1 $\mu \mathrm{M}$, at which it remains specific for PKA) (Kase et al., 1987) eliminated both attractive and repulsive responses to BDNF (Fig. 5c,d). Manipulation of cAMP level in neurons cultured on bound netrin-1 gradient generated similar results: low concentration of KT5720 (200 nM) caused repulsive axon initiation and turning, whereas high concentration of KT5720 (1 mM) resulted in seemingly random axon initiation and turning (supplemental Fig. S3, available at www.jneurosci. org as supplemental material). These results indicate that effectors of the cAMPPKA signaling pathway are not only modulatory in changing the direction of axon initiation and turning in the bound BDNF

angular distribution of the axon initiation site on the soma (Fig. $5 a$ ) and the final growth cone position (Fig. $5 b$ ) with respect to the direction of the gradient, for all polarized neurons exhibiting a single axon and multiple dendrites. On bound gradients generated with $2 \mu \mathrm{g} / \mathrm{ml}$ source BDNF, we observed only attractive response of the axon to BDNF (Fig. $6 a, b$ ). For neurons treated with function-blocking polyclonal antibodies against the highaffinity BDNF receptor TrkB, we found no preferred direction of axon initiation or turning on bound BDNF gradients (initiation $\mathrm{PI}=-0.02 \pm 0.02$; axon turning PI $=-0.02 \pm 0.02$ ). By further quantitative analysis of the BDNF density and gradient in the measured and simulated protein gradient profiles (Fig. 6), we concluded that the repulsive response was caused by the high level of BDNF action on the substrate.

The bidirectional polarization responses at regions of lowversus high-density bound BDNF resemble those found for growth cone turning in cultured Xenopus spinal neurons: In the same diffusible BDNF gradient, the growth cone may be switched from being attracted to being repelled toward the source of BDNF when the cAMP activity was reduced in the neuron (Song et al., 1997). We reasoned that exposure of these hippocampal neurons to the high-density bound BDNF may have resulted in a low-level cAMP activity. We thus further examined the axon initiation and turning in response to the bound BDNF after manipulations of gradient, but also required for these responses.

\section{Minimum effective gradient of bound BDNF for axon initiation and guidance}

To understand the long-range chemotropic function of axon guidance factors, it is important to know the minimal effective concentration or gradient of the factor. Using our diffusive printing gradient system, we have analyzed the angle of axon initiation and growth cone turning in each 100- $\mu \mathrm{m}$-wide region along the bound BDNF gradient produced with either 20 or $2 \mu \mathrm{g} / \mathrm{ml}$ source BDNF (Fig. 6a,b). The surface BDNF density (D) was estimated experimentally by measuring the fluorescent intensity of the immunostained gradient profile (Fig. $6 c, d$ ). From the simulated gradient profile, we calculated the absolute gradient as represented by the change in BDNF density $(\Delta \mathrm{D})$ over a $10 \mu \mathrm{m}$ distance ( $\sim$ width of the soma or growth cone), and relative gradient as depicted by the percentage change in the surface density $[(\Delta \mathrm{D}) /$ D] over a $10 \mu \mathrm{m}$ distance (Fig. $6 e, f$ ). Average angles of axon initiation and turning were measured from neurons with soma located at different regions along the bound BDNF gradient (Fig. $6 a, b)$, and were used to quantify the degree of attraction and repulsion of axons to the BDNF gradient. As shown in Figure 6, $a$ and $c$, attraction changed to repulsion when the BDNF density on the substrate was $\geq 160$ molecule $/ \mu \mathrm{m}^{2}$. 
We found that for BDNF gradients generated by diffusive printing, $\mathrm{D}$ and $\Delta \mathrm{D} / \mathrm{D}$ showed opposite monotonic changes with distance from the source, but $\Delta \mathrm{D}$ showed bell-shaped profiles that peaked at 200 and $400 \mu \mathrm{m}$ for the BDNF source concentration of 2 and $20 \mu \mathrm{g} / \mathrm{ml}$, respectively (Fig. 6e,f). In comparison with the profile of average angles for axon initiation and turning in both gradients (Fig. $6 a, b$ ), it is clear that the absolute change in density $(\Delta \mathrm{D})$ correlates better with neuronal responses. In other words, the absolute difference of BDNF-induced cellular activation across the neuron, rather than the mean or the relative changes in the activation level, is the dominant factor for determining the chemoattractive axon initiation and turning. On gradients created by both 2 and $20 \mu \mathrm{g} / \mathrm{ml}$ source $\mathrm{BDNF}$, the minimal $\Delta \mathrm{D}$ required for directional axon initiation and turning (Fig. $6 e, f$ ) was $1-2$ molecule $/ \mu \mathrm{m}^{2}$ across a $10 \mu \mathrm{m}$ distance. This corresponds to a difference of $\sim 25-50$ molecules between two halve surfaces of a $10 \times 10 \mu \mathrm{m}$ size growth cone, which is comparable to the minimal requirement of diffusive nerve growth factor (NGF) gradient for inducing neurite turning in a three-dimensional environment (Rosoff et al., 2004).

\section{Discussion}

We have developed a new "diffusive printing" technique for rapid production of stable microscopic gradients of substratebound proteins. Using this method, we found that bound gradients of netrin-1 and BDNF can polarize the axon initiation and turning in cultured hippocampal neurons. Furthermore, bound BDNF gradient caused attractive and repulsive polarizing response on gradients of low- and high-average density of BDNF, respectively. This bidirectional response to BDNF was shown to depend on the cytosolic cAMP level. Finally, our data showed that the neuron's attractive response to bound BDNF gradient depended on the absolute difference rather than the relative difference in the BDNF density across the neuron, with a minimal effective difference of $1-2$ BDNF molecule/ $\mu \mathrm{m}^{2}$ on the substrate surface.

When hippocampal neurons were plated on bound gradients of netrin-1 and low-density BDNF, neurites initiated on the side of the soma facing the high-protein density exhibited a much higher probability of differentiating into the axon than those facing the opposite side. This suggests that bound gradient of these factors are capable of triggering polarized axon differentiation, presumably through local activation of axon promoting factors in the neurite facing the high-density side and long-range inhibition of axon formation in all other neurites of the same neuron (Arimura and Kaibuchi, 2007). After the axon was formed, bound gradients of these factors could further polarize the cytoskeletal dynamics underlying growth cone extension by triggering a gradient of cytoplasmic second messengers, e.g., $\mathrm{Ca}^{2+}$ or cyclic nucleotides (Zheng and Poo, 2007), leading to chemo-
BDNF $(20 \mu \mathrm{g} / \mathrm{ml})$

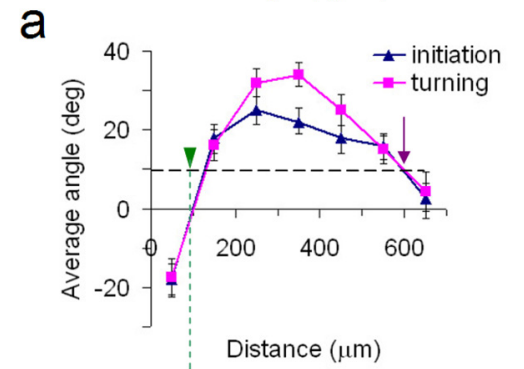

Distance $(\mu \mathrm{m})$

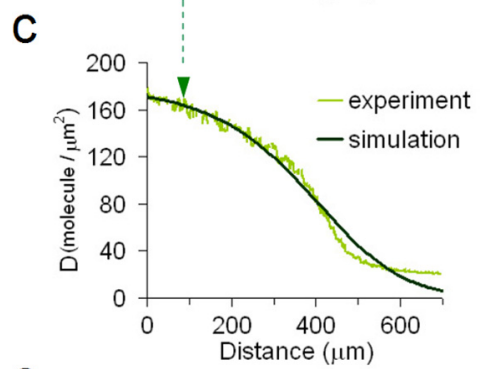

e

b

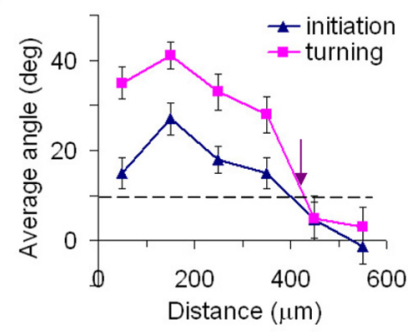

d

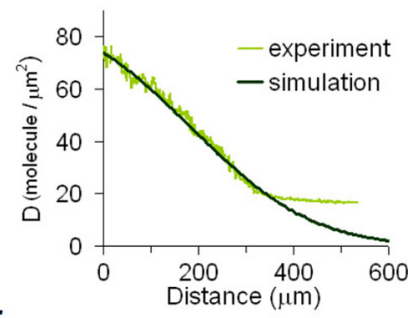

f

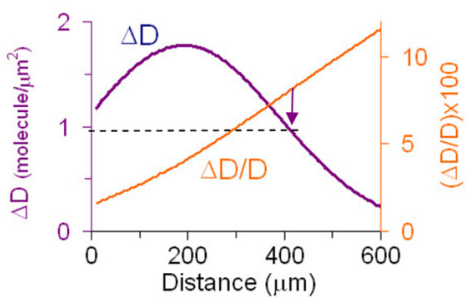

Figure 6. Quantitative analysis of axon initiation and turning on bound BDNF gradients. $\boldsymbol{a}, \boldsymbol{b}$, Average angles of axon initiation and turning for hippocampal neurons in $40 \mathrm{~h}$ culture on bound BDNF gradients created by 10 -min diffusive printing of BDNF at 20 traction to repulsion. $\boldsymbol{e}, \boldsymbol{f}$, Steepness of the substrate-bound BDNF gradients, as represented by the percentage $(\Delta D / D)$ and absolute $(\Delta D)$ change of surface density across every $10 \mu \mathrm{m}$ distance on simulated bound BDNF gradient created using 20 and 2 $\mu \mathrm{g} / \mathrm{ml}$ BDNF. Dashed lines and arrows mark the minimal $\Delta D$ required for detectable chemoattractive neuron response.

tropic turning of the growth cone toward the high-concentration side of the gradient. In both cases, signals triggered by the extracellular bound protein gradient are transduced and amplified into cytoplasmic gradients of cellular events, leading to polarized axon formation during the early phase of neuronal differentiation and directional turning of the growth cone after the axon is formed.

The turning of growth cones in response to the same diffusible gradient of axon guidance cues can be switched between attraction and repulsion, depending on the cytosolic concentration of cAMP, cGMP, and $\mathrm{Ca}^{2+}$ (Song et al. 1997; Gomez and Zheng, 2006; Nishiyama et al., 2003; Henley and Poo, 2004), but it remains unclear what in vivo factors may regulate the level of these secondary messengers. In vitro studies have shown that the axon's contact with extracellular laminin may result in a reduction of cytosolic cAMP level and the conversion of the growth cone's turning response from attraction to repulsion (Höpker et al., 1999; Joanne Wang et al., 2008). Our results here further demonstrate that such conversion via reduction of cAMP level could also be caused by the high concentration of the guidance factor itselfattractive at a distance from the source where the BDNF concentration is low, but repulsive when the axon reaches the region of high BDNF concentration. The bidirectional responses toward the gradient of the same guidance factor may serve as a potential 
mechanism that allows an axon to be first attracted to a guidepost cell and then be repelled from it for the next target. Such concentration-dependent bidirectional responses may also be relevant for the action of $\mathrm{BDNF}$ on synaptic transmission and plasticity (Poo, 2001). The cytoplasmic mechanisms underlying these bidirectional responses to BDNF remain to be further elucidated. The effects of low-level KT5720 and Sp-Br-cAMP (Fig. $5 c, d)$ suggest that the high-level BDNF signaling may result in a reduction of cytosolic cAMP. However, Gao et al. (2003) showed that in cerebellar and DRG neurons BDNF incubation increased the cAMP level by inhibiting phosphodiesterase PDE4. The reduction of cAMP at high-level BDNF activation could result from the TrkB internalization triggered by BDNF binding (Sommerfeld et al. 2000), although at this stage it is unknown whether bound BDNF is effective in triggering TrkB internalization. Furthermore, bound extracellular BDNF gradient may differ from the diffusible BDNF gradient established in solution by its immobilizing action on TrkB receptors in the plasma membrane. Such action may affect the formation of receptor-associated membrane microdomains that influence axon initiation and turning (Guirland et al., 2004). Further study is required to elucidate the mechanisms underlying ligand concentration-dependent polarization and the effects of ligand-receptor immobilization in the establishment and amplification of polarized cytoplasmic signals. Further in vivo investigation on how BDNF regulate cAMP level will also contribute to the development of therapies for spinal cord injury (Neumann et al., 2002; Qiu et al., 2002).

The diffusive printing method mimics the natural process of protein secretion, diffusion and adsorption; thus, the resultant gradient profile simulates naturally formed bound protein gradients in vivo. In contrast to the exponential gradient produced by the diffusion alone, where the relative concentration gradient $(\Delta \mathrm{C} / \mathrm{C})$ is fixed and the absolute gradient $(\Delta \mathrm{C})$ decays exponentially with decreasing concentration, our analysis demonstrates that a bound gradient generated by the combined effect of diffusion and surface adsorption has steeper gradients at lower concentrations (Fig. 6e,f), a theoretically predicted property of the optimal gradient for chemotactic action over the maximum possible distance (Goodhill and Urbach, 1999). Additionally, neuronal responses to guidance factors, as exemplified by BDNF, may not be uniform along such a gradient but exhibits a maximum over a region with the highest absolute gradient, allowing most effective guidance action of the factor on neurons within a specific distance from the secretion site.

Compared with previous methods for producing linear gradients of bound proteins (Baier and Bonhoeffer, 1992; Dertinger et al., 2002; Moore et al., 2006; von Philipsborn et al., 2006; Smith et al., 2004; Liu et al., 2007) in studies on neuronal development, our diffusive printing method uses a substantially simpler system set-up and requires a much shorter preparation time. Notably, only small amounts of protein are required to produce a gradient, making it practical to study proteins of limited quantity. This system also allows conventional cell culture and imaging on glass coverslips, with minimal problems of sheer stress, metabolite exchange, cytotoxicity, or optical interference. All these advantages allow fast screening and quantitative analysis of neuronal development in a stable and precisely controlled in vitro gradient environment.

\section{References}

Adler CE, Fetter RD, Bargmann CI (2006) UNC-6/Netrin induces neuronal asymmetry and defines the site of axon formation. Nat Neurosci 9:511-518.
Arimura N, Kaibuchi K (2007) Neuronal polarity: from extracellular signals to intracellular mechanisms. Nat Rev Neurosci 8:194-205.

Baier H, Bonhoeffer F (1992) Axon guidance by gradients of a targetderived component. Science 255:472-475.

de la Torre JR, Höpker VH, Ming, GL, Poo, MM, Tessier-Lavigne M, Hemmati-Brivanlou A, Holt CE (1997) Turning of retinal growth cones in a netrin-1 gradient mediated by the netrin receptor DCC. Neuron 19:1211-1224.

Dertinger SK, Jiang X, Li Z, Murthy VN, Whitesides GM (2002) Gradients of substrate-bound laminin orient axonal specification of neurons. Proc Natl Acad Sci U S A 99:12542-12547.

Dickson BJ (2002) Molecular mechanisms of axon guidance. Science 298:1959-1964.

Dotti CG, Sullivan CA, Banker GA (1988) The establishment of polarity by hippocampal-neurons in culture. J Neurosci 8:1454-1468.

Gao Y, Nikulina E, Mellado W, Filbin MT (2003) Neurotrophins elevate cAMP to reach a threshold required to overcome inhibition by MAG through extracellular signal-regulated kinase-dependent inhibition of phosphodiesterase. J Neurosci 23:11770-11777.

Genç B, Ozdinler PH, Mendoza AE, Erzurumlu RS (2004) A chemoattractant role for NT-3 in proprioceptive axon guidance. PLoS Biology 2:e403.

Genzer J, Bhat RR (2008) Surface-bound soft matter gradients. Langmuir 24:2294-2317.

Gillespie LN (2003) Regulation of axonal growth and guidance by the neurotrophin family of neurotrophic factors. Clin Exp Pharmacol Physiol 30:724-733.

Goldman RD, Spector DL (2004) Live cell imaging: a laboratory manual, pp 270-271. New York: Cold Spring Harbor Laboratory.

Gomez TM, Zheng JQ (2006) The molecular basis for calcium-dependent axon pathfinding. Nat Rev Neurosci 7:115-125.

Goodhill GJ, Urbach JS (1999) Theoretical analysis of gradient detection by growth cones. J Neurobiol 41:230-241.

Guirland C, Suzuki S, Kojima M, Lu B, Zheng JQ (2004) Lipid rafts mediate chemotropic guidance of nerve growth cones. Neuron 42:51-62.

Gundersen RW, Barrett JN (1980) Characterization of the turning response of dorsal root neurites toward nerve growth-factor. J Cell Biol 87:546-554.

Henley J, Poo MM (2004) Guiding neuronal growth cones using Ca2+ signals. Trends Cell Biol 14:320-330.

Höpker VH, Shewan D, Tessier-Lavigne M, Poo M, Holt C (1999) Growthcone attraction to netrin- 1 is converted to repulsion by laminin-1. Nature 401:69-73.

Joanne Wang C, Li X, Lin B, Shim S, Ming GL, Levchenko A (2008) A microfluidics-based turning assay reveals complex growth cone responses to integrated gradients of substrate-bound ECM molecules and diffusible guidance cues. Lab Chip 8:227-237.

Jones JI, Gockerman A, Busby WH Jr, Camacho-Hubner C, Clemmons DR (1993) Extracellular-matrix contains insulin-like growth factor binding protein-5: potentiation of the effects of IGF-I. J Cell Biol 121:679-687.

Kase H, Iwahashi K, Nakanishi S, Matsuda Y, Yamada K, Takahashi M, Murakata C, Sato A, Kaneko M (1987) K-252 compounds, novel and potent inhibitors of protein-kinase-c and cyclic nucleotide-dependent proteinkinases. Biochem Biophys Res Commun 142:436-440.

Keenan TM, Folch A (2008) Biomolecular gradients in cell culture systems. Lab Chip 8:34-57.

Kennedy TE, Wang H, Marshall W, Tessier-Lavigne M (2006) Axon guidance by diffusible chemoattractants: a gradient of netrin protein in the developing spinal cord. J Neurosci 26:8866-8874.

Liu LY, Ratner BD, Sage EH, Jiang S (2007) Endothelial cell migration on surface-density gradients of fibronectin, VEGF, or both proteins. Langmuir 23:11168-11173.

Lohof AM, Quillan M, Dan Y, Poo MM (1992) Asymmetric modulation of cytosolic camp activity induces growth cone turning. J Neurosci 12:1253-1261.

Mateo C, Abian O, Fernandez-Lafuente R, Guisan JM (2000a) Increase in conformational stability of enzymes immobilized on epoxy-activated supports by favoring additional multipoint covalent attachment. Enzyme and Microbial Technology 26:509-515.

Mateo C, Fernández-Lorente G, Abian O, Fernández-Lafuente R, Guisán JM (2000b) Multifunctional epoxy supports: A new tool to improve the covalent immobilization of proteins. The promotion of physical adsorp- 
tions of proteins on the supports before their covalent linkage. Biomacromolecules 1:739-745.

Ming GL, Song HJ, Berninger B, Holt CE, Tessier-Lavigne M, Poo MM (1997) cAMP-dependent growth cone guidance by netrin-1. Neuron 19:1225-1235.

Moore K, MacSween M, Shoichet M (2006) Immobilized concentration gradients of neurotrophic factors guide neurite outgrowth of primary neurons in macroporous scaffolds. Tissue Eng 12:267-278.

Nathan C, Sporn M (1991) Cytokines in context. J Cell Biol 113:981-986.

Neumann S, Bradke F, Tessier-Lavigne M, Basbaum AI (2002) Regeneration of sensory axons within the injured spinal cord induced by intraganglionic cAMP elevation. Neuron 34:885-893.

Nishiyama M, Hoshino A, Tsai L, Henley JR, Goshima Y, Tessier-Lavigne M, Poo MM, Hong KS (2003) Cyclic AMP/GMP-dependent modulation of $\mathrm{Ca} 2+$ channels sets the polarity of nerve growth-cone turning. Nature 423:990-995.

Park JE, Keller GA, Ferrara N (1993) Vascular endothelial growth factor (VEGF) isoforms: differential deposition into the subepithelial extracellular-matrix and bioactivity of extracellular matrix-bound VEGF. Molecular Biology of the Cell 4:1317-1326.

Poo MM (2001) Neurotrophins as synaptic modulators. Nat Rev Neurosci 2:24-32.

Qiu J, Cai D, Dai H, McAtee M, Hoffman PN, Bregman BS, Filbin MT (2002) Spinal axon regeneration induced by elevation of cyclic AMP. Neuron 34:895-903.

Rosoff WJ, Urbach JS, Esrick MA, McAllister RG, Richards LJ, Goodhill GJ (2004) A new chemotaxis assay shows the extreme sensitivity of axons to molecular gradients. Nat Neurosci 7:678-682.
Saksela O, Moscatelli D, Sommer A, Rifkin DB (1988) Endothelial cellderived heparan-sulfate binds basic fibroblast growth-factor and protects it from proteolytic degradation. J Cell Biol 107:743-751.

Serafini T, Colamarino SA, Leonardo ED, Wang H, Beddington R, Skarnes WC, Tessier-Lavigne M (1996) Netrin-1 is required for commissural axon guidance in the developing vertebrate nervous system. Cell 87:1001-1014.

Smith JT, Tomfohr JK, Wells MC, Beebe TP Jr, Kepler TB, Reichert WM (2004) Measurement of cell migration on surface-bound fibronectin gradients. Langmuir 20:8279-8286.

Sommerfeld MT, Schweigreiter R, Barde YA, Hoppe E (2000) Downregulation of the neurotrophin receptor TrkB following ligand binding. Evidence for an involvement of the proteasome and differential regulation of TrkA and TrkB. J Biol Chem 275:8982-8990.

Song H, Poo M (2001) The cell biology of neuronal navigation. Nature Cell Biology 3:E81-E88.

Song HJ, Ming GL, Poo MM (1997) CAMP-induced switching in turning direction of nerve growth cones. Nature 388:275-279.

Tessier-Lavigne M, Goodman CS (1996) The molecular biology of axon guidance. Science 274:1123-1133.

Tessier-Lavigne M, Placzek M, Lumsden AG, Dodd J, Jessell TM (1988) Chemotropic guidance of developing axons in the mammalian central nervous system. Nature 336:775-778.

von Philipsborn AC, Lang S, Loeschinger J, Bernard A, David C, Lehnert D, Bonhoeffer F, Bastmeyer M (2006) Growth cone navigation in substrate-bound ephrin gradients. Development 133:2487-2495.

Zheng JQ, Poo MM (2007) Calcium signaling and neuronal motility. Annu Rev Cell Dev Biol 23:375-404. 